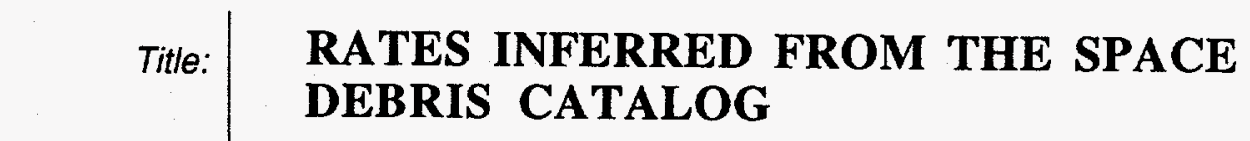

Author(s):

Submitted to:

Gregory H. Canavan, DDP

For discussions outside the Laboratory

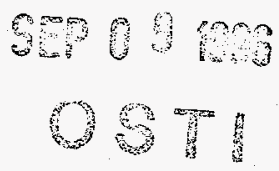

Date: August 1996
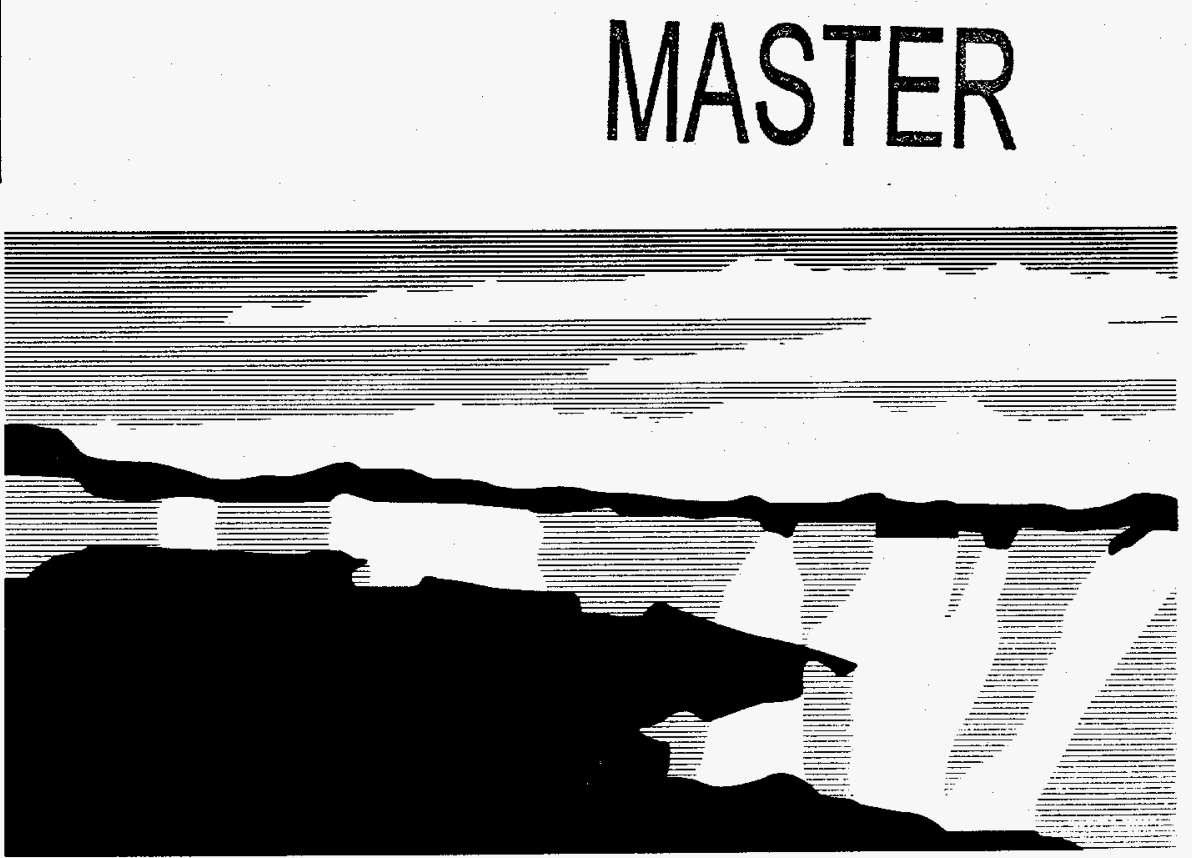

NATIONAL LABORATORY

Los Alamos National Laboratory, an affirmative action/equal opportunity employer, is operated by the University of California for the U.S. Department of Energy under contract W-7405-ENG-36. By acceptance of this article, the publisher recognizes that the U.S. Government retains a nonexclusive, royalty-free license to publish or reproduce the published form of this contribution, or to allow others to do so, for U.S. Government purposes. The Los Alamos National Laboratory requests that the publisher identify this article as work performed under the auspices of the U.S. Department of Energy. 


\section{DISCLAIMER}

Portions of this document may be illegible in electronic image products. Images are produced from the best available original document. 


\title{
RATES INFERRED FROM THE SPACE DEBRIS CATALOG
}

\author{
Gregory H. Canavan
}

\begin{abstract}
Collision and fragmentation rates are inferred from the AFSPC space debris catalog and compare with estimates from other treatments. The collision rate is evaluated without approximation. The fragmentation rate requires additional empirical assessments. The number of fragments per collision is low compared to analytic and numerical treatments, is peaked low, and falls rapidly with altitude.
\end{abstract}

is

This note discusses the inference of collision and fragment production rates from the AFSPC space debris catalog. It calculates collision and fragment production rates and compares them with estimates from other treatments. The catalog can be sorted on a desk computer to produce object densities as a function of area and altitude. The distribution is irregular and has several prominent peaks, but that does not affect the low-order statistics treated here. The results show some signs of incompleteness for objects smaller than about $0.1 \mathrm{~m}^{2}$. The LEO distributions group into low, medium, and high altitude bands, which contain predominantly large, medium, and small objects.

The catalog collision frequency can be formulated analytically and solved without approximation. It is small below $550 \mathrm{~km}$, but rises sharply with altitude. Contribution from the large objects at low altitudes is suppressed; the contribution from the maximum at $1450 \mathrm{~km}$ is a small and could be ignored were it not for the long decay lifetimes of objects at those altitudes.

Evaluating fragment production rates requires the introduction of a factor to account for the number of fragments produced in each collision, which has been measured from a series of DoD/DNA experiments, in a form suitable for inclusion. The dominant term can be extracted and treated simply through a combination of analytic and numerical evaluations. The fragment production function resembles the collision rate, except that its heavier area weighting penetrates down to about $350 \mathrm{~km}$. It integrates to about 2.4 fragments $/ \mathrm{yr}$ by $1050 \mathrm{~km}$, which is about $90 \%$ of its asymptotic value.

The ratio of the number of fragments per year to the number of collisions per year gives the average number of fragments per collision, which is about 53-slightly smaller than the 80 predicted by analytic calculations and, but well below the 480 from other estimates. The production rate is peaked at low altitudes. If the large objects below $500 \mathrm{~km}$ were eliminated, the average number of fragments per collision would drop to about 40 . The cascade coefficient falls with altitude; at $950 \mathrm{~km}$ the cascade doubling time would be about 1100 years. At higher altitudes the cascade coefficient falls a further factor of 3 , so the cascade times would be longer. Overall, the insights into collision, fragmentation, and cascade from the catalog are consistent with those gained from analytic models using only low order moments of it. 
Object density. Sorting the AFSPC ' 95 catalogue by finding all particles in a given altitude interval $\mathrm{dz}$ at $\mathrm{z}$ with areas larger than a given area $\mathrm{A}$ produces a set of cumulative densities

$$
\mathrm{N}(\mathrm{z}, \mathrm{A})=\int_{\mathrm{A}} \mathrm{dA}^{\prime} \mathrm{n}\left(\mathrm{z}, \mathrm{A}^{\prime}\right) \text {, }
$$

The upper limit of Eq. (1) is the area at which the distribution falls sharply, about $100 \mathrm{~m}^{2}$. The lower limit is of concern here. The object density $n$ can be found by differentiating Eq. (1)

$$
\mathrm{n}(\mathrm{z}, \mathrm{A})=-\partial \mathrm{N}(\mathrm{z}, \mathrm{A}) / \partial \mathrm{A} \text {, }
$$

which is shown in Fig. 1. The density $n$ has large peaks over wide ranges of object areas at 950 and $1450 \mathrm{~km}$. It also has sharp spikes at $0.3,3$, and $30 \mathrm{~m}^{2}$ at those altitudes, which represent intact objects, as well as broad distributions that represent debris. The sorting was performed with a resolution about an order of magnitude finer than that shown in Fig. 1, which is filtered to present object density in logarithmically spaced bins so that the contents of each bin can be summed to see the relative contributions to the density at each altitude. Barely visible in the figure at about $400 \mathrm{~km}$ are several large objects that add little to the density but contribute significantly to the area and collision rates calculated below. Just visible in the figure is the fact that the object numbers fall for all altitudes as $A$ decreases below about $0.1 \mathrm{~m}^{2}$. While this could be an indication that the sorting was not sufficiently accurate for objects at 0.01 and $0.03 \mathrm{~m}^{2}$, it is also possible that it could reflect incomplete measurement of small objects in the Catalog.

As the densities for all altitudes become needlessly complicated when they are all treated together, the paragraphs below discuss the size distribution of objects in the 300-700,700-1100, and 1100-1600 altitude bands separately before drawing overall conclusions. Figure 2 shows the object densities in $100 \mathrm{~km}$ altitude bands centered on $350,450,550$, and $650 \mathrm{~km}$. There is at most a few tens of objects in each area interval. For small areas, the numbers increase monotonically with altitude, as the smaller objects decay rapidly at lower altitudes. Each bin is a factor of three larger than the prior one up to $10 \mathrm{~m}^{2}$, after which the intervals drop down to about $10 \%$ of the area for greater accuracy in counting the objects that contain most of the area. The distributions are relatively flat out to about $10 \mathrm{~m}^{2}$, after which they fall rapidly, although it is difficult to infer the slope from this bin structure. The objects at $350 \mathrm{~km}$ begin to fall at about $3 \mathrm{~m}^{2}$; the objects at 450 and higher $\mathrm{km}$ begin to fall at about $10 \mathrm{~m}^{2}$. However, there are a few larger objects at 550 and 650 $\mathrm{km}$ with areas of about $100 \mathrm{~m}^{2}$; an object that divides its time between 350 and $450 \mathrm{~km}$ with an area of about $700 \mathrm{~m}^{2}$, and an object at $350 \mathrm{~km}$ with an area of $900 \mathrm{~m}^{2}$.

For $\mathrm{z}>500 \mathrm{~km}$, there is a density of about 1 to 10 objects per $100 \mathrm{~km}$ per area interval. For areas $>20-30 \mathrm{~m}^{2}$, the densities drop to the individual objects cited above. The distribution also shows a large number of objects in the $25-55 \mathrm{~m}^{2}$ interval that correspond to 0.5 to $5 \%$ of an object, which represent the small projections of geosynchronous transfer orbit stages and Molniya orbits into LEO.

Figure 3 shows the object distribution for $700-1100 \mathrm{~km}$, which resembles Fig. 2, but is 
about an order of magnitude higher below $10 \mathrm{~m}^{2}$, where, the densities at the different altitudes are quite close, apart from the peak at $3 \mathrm{~m}^{2}$ and $950 \mathrm{~km}$ already noted in Fig. 1. Again, the numbers of objects fall rapidly above 3-10 $10 \mathrm{~m}^{2}$, and there are a number of partial projections of eccentric orbits at $25-55 \mathrm{~m}^{2}$, although in this altitude range they are due to less eccentric orbits. However, in contrast to Fig. 2 there are essentially no objects larger than $60 \mathrm{~m}^{2}$ in this altitude interval.

Figure 4 Figure 4 shows the object distribution for $1100-1600 \mathrm{~km}$, which resembles Fig. 2, but falls off somewhat faster, apart from the peak at $0.3 \mathrm{~m}^{2}$ at $1450 \mathrm{~km}$, which is more prominent in the linear scale of Fig. 1. It is about an order of magnitude higher below $10 \mathrm{~m}^{2}$, where, the densities at the different altitudes are quite close, apart from the peak at $3 \mathrm{~m}^{2}$ and 950 $\mathrm{km}$ already noted in Fig. 1. In contrast to Figs. 2 and 3 there are essentially no objects larger than $35 \mathrm{~m}^{2}$ in this altitude interval, so the falloff is very sharp.

Figure 5 combines the three previous figures into a plot of the number of objects in the various bins and the cumulative number of objects up to a given area, integrated over all altitudes. The $\approx 1,000$ objects per area bin at small A largely come from the hundreds of objects per bin in the $700-1100 \mathrm{~km}$ altitude range from Fig. 3. The $50-100$ and $700-900 \mathrm{~m}^{2}$ objects come from $300-$ $700 \mathrm{~km}$. The $0.3 \mathrm{~m}^{2}$ spike at $1450 \mathrm{~km}$ is largely masked in the integration over altitudes. The top curve for the cumulative number of particles increases slowly, reaching $92 \%$ of its asymptotic value of 5270 objects by $3 \mathrm{~m}^{2}$. Figure 6 shows the differential area in the bin at area $A$, which is $\mathrm{n}(\mathrm{z}, \mathrm{A}) \mathrm{A}$, and the cumulative area up to $\mathrm{A}$, which is

$$
\Sigma(\mathrm{z}, \mathrm{A})=\int_{\mathrm{C}^{\mathrm{A}}} \mathrm{dA}^{\prime} \mathrm{n}\left(\mathrm{z}, \mathrm{A}^{\prime}\right) \mathrm{A}^{\prime} \text {; }
$$

where $C \approx 0.1 \mathrm{~m}^{2}$ is the area of the smallest objects in the catalogue. The differential area $n(z, A) A$ increases roughly as $A$ for small objects, peaks at about $7,000 \mathrm{~m}^{2}$ at $A \approx 3 \mathrm{~m}^{2}$, and then falls sharply to $\approx 100 \mathrm{~m}^{2}$ at $\approx 30 \mathrm{~m}^{2}$. The level of support there is due to the series of intact objects in that area range in Fig. 5, whose unitary contributions to $\mathrm{n}$ give contributions to $\mathrm{n}(\mathrm{z}, \mathrm{A}) \mathrm{A}$ proportional to $A$, as seen. Thus, it is not fortuitous that the large objects lie along the same line. The top curve for $\Sigma(\mathrm{z}, \mathrm{A})$ increases with $\mathrm{nA}$ for $\mathrm{A}$ small, but only reaches about $50 \%$ of its asymptotic value by $3 \mathrm{~m}^{2}$. It reaches $90 \%$ of its asymptotic value of $18,500 \mathrm{~m}^{2}$ by $A=100 \mathrm{~m}^{2}$, but the two large objects at $700-900 \mathrm{~m}^{2}$ still make a $10 \%$ contribution.

The collision rate at altitude $\mathrm{z}$ is given by the integral

$$
\mathrm{R}=1 / 2 \int_{\mathrm{V}} \mathrm{dV} \int_{\mathrm{A}} \mathrm{dA} \int_{\mathrm{B}} \mathrm{dB}(\mathrm{n}(\mathrm{z}, \mathrm{A}) / \mathrm{W})(\mathrm{n}(\mathrm{z}, \mathrm{B}) / \mathrm{W})(\mathrm{A}+\mathrm{B}) \mathrm{v} \text {, }
$$

where $A$ and $B$ are the areas of the colliding objects, $v$ is their relative velocity,

$$
\mathrm{W}=4 \pi\left(\mathrm{R}_{e}+\mathrm{z}\right)^{2} \mathrm{dz}
$$

is the volume of the shell of thickness $\mathrm{dz}=100 \mathrm{~km}$ at $\mathrm{z}$ within which they interact, and $\mathrm{R}_{\mathrm{e}} \approx$ $6,400 \mathrm{~km}$. Their relative velocity is $\mathrm{v} \approx 10 \mathrm{~km} / \mathrm{s}$, although the effective velocity is increased about $25 \%$ by the current mix of inclinations of satellites. ${ }^{1}$ By symmetry, $R$ reduces to

$$
R=v / W^{2} \int_{V} d V \int_{A} d A \int_{B} d B n(z, A) n(z, B) A
$$




$$
\begin{aligned}
& =(\mathrm{v} / \mathrm{W}) \int_{\mathrm{A}} \mathrm{dA} \mathrm{n}(\mathrm{z}, \mathrm{A}) \mathrm{A} \int_{\mathrm{B}} \mathrm{dB} \mathrm{n}(\mathrm{z}, \mathrm{B}) \\
& =(\mathrm{v} / \mathrm{W}) \sum(\mathrm{z}, \mathrm{C}) \mathrm{N}(\mathrm{z}, \mathrm{C}),
\end{aligned}
$$

which is the product of the total number $\mathrm{N}(\mathrm{z}, \mathrm{C})$, and area, $\Sigma(\mathrm{z}, \mathrm{C})$, of catalog objects, which involves only quantities determined by the sorting above. In particular, $N(z, C)$ and $\Sigma(\mathrm{z}, \mathrm{C})$ depend primarily on the number of particles and area, rather than the complex details of their distribution shown in Fig. 1. Figure 7 shows $N(z, C)$ and $\Sigma(z, C)$ as functions of altitude $z$. The bottom curve is the total number of objects at each altitude, a low moment of the AFSPC Catalog, which has pronounced maxima at about 950 and $1450 \mathrm{~km}$.

The top curve is the cumulative area at each altitude, $\Sigma(\mathrm{z}, \mathrm{C})$, which is broadly similar to $\mathrm{N}(\mathrm{z}, \mathrm{C})$. The peaks at 350,950 , and $1450 \mathrm{~km}$ correspond to the concentrations of large, medium, and small satellites discussed above. The ratio of $\sum(\mathrm{z}, \mathrm{C})$ to $\mathrm{N}(\mathrm{z}, \mathrm{C})$ in the interval 450 to $850 \mathrm{~km}$ is larger than that in 1050 to 1350 because of the larger fraction of larger satellites there, indicating the presence of significant numbers of large particles in the decaying spectrum. The effect diminishes above $1600 \mathrm{~km}$, where most of the objects are debris.

Fig 8 shows the collision rate at each altitude $\mathrm{R}(\mathrm{z})=(\mathrm{v} / \mathrm{W}) \sum(\mathrm{z}, \mathrm{C}) \mathrm{N}(\mathrm{z}, \mathrm{C})$, and the total collision rate $\int \mathrm{dz} R(\mathrm{z})$ integrated over all altitudes up to $2000 \mathrm{~km}$. The bottom curve for $\mathrm{R}$ is small for $\mathrm{z}<550 \mathrm{~km}$; the multiplication of $\Sigma(\mathrm{z}, \mathrm{C})$ by the catalog $\mathrm{N}(\mathrm{z}, \mathrm{C})$, which is small there, leaves little residue of the large peak in $\Sigma(\mathrm{z}, \mathrm{C})$ at $450 \mathrm{~km}$. The peak at $950 \mathrm{~km}$ persists, but the relative magnitude of the peak at $1450 \mathrm{~km}$ is further diminished. Overall, there is a roughly triangular $R$ increasing from $400 \mathrm{~km}$ to about $950 \mathrm{~km}$, together with a smaller symmetric triangle at $1450 \mathrm{~km}$.

The total collision rate is also small below $550 \mathrm{~km}$, but rises sharply to about $0.044 / \mathrm{yr}$ by $1050 \mathrm{~km}$, which is $85 \%$ of its asymptotic value of $0.051 / \mathrm{yr}$. The contribution from the large objects at low altitudes is suppressed, and the contribution from the maximum at $1450 \mathrm{~km}$ is roughly a $15 \%$ effect, which could be ignored to first order were it not for the long decay lifetimes of objects at those altitudes.

The particle production rate $Q$ introduces an additional factor in the kernel of Eq. (6) to account for the number of additional fragments produced in each collision. The factor is usually expressed as $B^{\prime}(Z / m)^{B}$ in terms of the total mass of the colliding particles, $Z$, the mass of the projectile, $\mathrm{m}$, and an experimentally determined production exponent, $\mathrm{B} \approx 0.6$. The other factor $\mathrm{B}^{\prime}$ $=1 / \mathrm{B}-1 \approx 0.67$, is a normalization constant determined by the conservation of mass. ${ }^{2}$ For $\mathrm{B} \approx$ 0.6 and $\mathrm{Z} / \mathrm{m}=1,000$, the greatest discrepancy between the impactor and target object masses allowed by JSC's collision limit, 3 this scaling gives $\approx 0.67 \times 1000^{0.6} \approx 42$ particles.

It is necessary to reformulate these arguments in terms of object areas rather than masses. If it is assumed 4 that $\mathrm{A}=\mathrm{Km}^{\mathrm{s}}$, or $\mathrm{m}=(\mathrm{A} / \mathrm{K})^{1 / \mathrm{s}}$, the extra factor in the collision kernel representing: the production of particles as large as those in the catalog becomes $\mathrm{B}^{\prime}\left[(\mathrm{A} / \mathrm{K})^{1 / \mathrm{s}} /(\mathrm{C} / \mathrm{K})^{1 / s}\right]^{B}=$ $(\mathrm{A} / \mathrm{C})^{\mathrm{B} / \mathrm{s}}$,so the particle production rate becomes 


$$
Q=v / 2 W^{2} \int V d V \int d A \int d B n(z, A) n(z, B) B^{\prime}(A+B)^{1+B / s / C B / s} .
$$

This equation can be evaluated directly, but the dominant term in the kernel is 5

$$
\begin{aligned}
& \mathrm{Q} \approx \mathrm{vB}^{\prime} / \mathrm{WC}^{\mathrm{B} / \mathrm{s}} \int \mathrm{dA} \int \mathrm{dB} \mathrm{n}(\mathrm{z}, \mathrm{A}) \mathrm{n}(\mathrm{z}, \mathrm{B}) \mathrm{A}^{1+\mathrm{B} / \mathrm{s}} \\
& \approx \mathrm{vB}^{\prime} / \mathrm{wC}^{\mathrm{B} / \mathrm{s}} \int \mathrm{dA} n(\mathrm{z}, \mathrm{A}) \mathrm{A}^{1+\mathrm{B} / \mathrm{s}} \int \mathrm{dB} n(\mathrm{z}, \mathrm{B}) \\
& \approx v B^{\prime} / W^{B / s} N(z, C) \int d A n(z, A) A^{1+B / s} \text {, }
\end{aligned}
$$

which is the product of the density, a moment of the area distribution intermediate between the 1st and 2nd, and some normalizing constants. For $\mathrm{C}=0.01 \mathrm{~m}^{2}, \mathrm{~B}=0.6$, and $\mathrm{s}=1$, i.e., object areas proportional to mass, $\mathrm{B}^{\prime} / \mathrm{C}^{\mathrm{B} / \mathrm{s}} \approx(1 / 0.6-1) / 0.01^{0.6} \approx 10.6$. In performing the integral $\int \mathrm{dB}$ $\mathrm{n}(\mathrm{z}, \mathrm{B})$, the condition that the impactor mass should be within a factor of 1000 of the target should be imposed, but the catalog is so lacking in objects larger than $100 \mathrm{~m}^{2}$, so the condition would only apply to objects smaller than $0.1 \mathrm{~m}^{2}$, which are present in modest numbers as noted above. Thus, the condition can be ignored with little impact for this estimate.

Figure 9 shows the fragment production rate as a function of altitude along with its cumulative value up to each altitude. The function $Q$ resembles the differential collision rate $R$ of Fig. 8, except that its heavier area weighting produces a penetration down to about $350 \mathrm{~km}$ where some large objects lie. That also produces a slight minimum in production at about $500 \mathrm{~km}$. Q integrates to about $2.4 / \mathrm{yr}$ by $1050 \mathrm{~km}$, which is about $90 \%$ of its asymptotic value of $\approx 2.7 / \mathrm{yr}$. The increment at $1450 \mathrm{~km}$ is a small effect, because most of the objects there are small.

Figure 10 shows the ratio of $Q$ to $R$ as a function of altitude. As $Q$ gives the number of fragments produced per year and $\mathrm{R}$ gives the number of collisions per year, their ratio gives the average number of fragments per catalog collision. The average number is about 53 , which is somewhat lower than the 80 fragments per collision predicted by analytic calculations ${ }^{6}$ and well below the 480 fragments per collision from other estimates. ${ }^{7}$ The figure also indicates that $Q$ is peaked at low altitudes, where the large objects lied. If they were eliminated from the sort, the fragment production there would drop significantly. However, the orbital decay times there are sufficiently short that any debris produced would be removed quickly. If the peaks below $500 \mathrm{~km}$ were eliminated, the average number of fragments per collision would drop to about 40 .

Figure 11 shows the cascade coefficient, which is the ratio of the fragment production rate $\mathrm{Q}$ to the square of the density at each altitude. The two sets of points represent two ways of avoiding self-collisions between large objects, so their agreement gives some indication of the sensitivity of the numerics. It measures the relative rate of production of fragments by cascading at each altitude. It has a peak of about 0.0002 fragment/yr-object ${ }^{2}$ at $350 \mathrm{~km}$. There are about 30 objects in the $100 \mathrm{~km}$ bin there, so the actual production rate is about $0.0002 \times 30^{2} \approx 0.18 / \mathrm{yr}$. The doubling time due to collisions is $\approx 30 / 0.18 / \mathrm{yr} \approx 170$ years, which is long compared to orbital decay there, which takes months. The cascade coefficient falls with altitude to slightly under $10^{-6}$ fragment/yr-object ${ }^{2}$ at $950 \mathrm{~km}$, where the cascade doubling time would be $\approx 1 / 890$ objects $\times 10^{-6}$ 
fragment/yr-object ${ }^{2} \approx 1100$ years. At higher altitudes the cascade coefficient falls a further factor of 3 , so the cascade times would be longer.

Summary and conclusions. This note evaluates collision and fragment production rates from the AFSPC space debris catalog and compares them with estimates from other treatments. The catalog can be sorted on a desk computer to produce object densities as a function of area and altitude. The distribution is irregular and has several prominent peaks, but that does not affect the relatively low-order statistics treated here. The results show some signs of incompleteness for objects smaller than about $0.1 \mathrm{~m}^{2}$. The LEO distributions group into low, medium, and high altitude bands, which contain predominantly large, medium, and small objects, respectively. The bands also differ markedly in the cutoff size beyond which there are no larger objects.

The evaluation of the catalog collision frequency can be formulated analytically and solved without approximation. The collision rate is also small below $550 \mathrm{~km}$, but rises sharply with altitude to about $80-90 \%$ of its asymptotic value of $0.051 / \mathrm{yr}$ by about $1050 \mathrm{~km}$. In its evaluation, the contribution from the large objects at low altitudes is suppressed, and the contribution from the maximum at $1450 \mathrm{~km}$ is a $15 \%$ effect, which could be ignored to first order were it not for the long decay lifetimes of objects at those altitudes.

Evaluating the particle production rate requires the introduction of an additional factor in the kernel of the collision integral to account for the number of additional fragments produced in each collision. The factor has been determined roughly from a series of DoD/DNA experiments, which have measured it experimentally in a form suitable for inclusion. The resulting equation can be evaluated directly, but it is has been shown elsewhere that the dominant term in the kernel can be extracted and treated simply through a combination of analytic and numerical evaluations. The fragment production function resembles the collision rate, except that its heavier area weighting penetrates down to about $350 \mathrm{~km}$. It integrates to about 2.4 fragments/yr by $1050 \mathrm{~km}$, which is about $90 \%$ of its asymptotic value. The increment from $1450 \mathrm{~km}$ is small.

The ratio of the number of fragments per year to the number of collisions per year gives the average number of fragments per collision, which is about 53-slightly smaller than the 80 predicted by analytic calculations and well below the 480 from other estimates. The production rate is peaked at low. If the large objects below $500 \mathrm{~km}$ were eliminated, the average number of fragments per collision would drop to about 40 . The cascade coefficient has a peak at $350 \mathrm{~km}$, but there are few objects there to cascade. The cascade coefficient falls with altitude; at $950 \mathrm{~km}$ the cascade doubling time would be about 1100 years. At higher altitudes the cascade coefficient falls a further factor of 3, so the cascade times would be longer. Overall, the insights into collision, fragmentation, and cascade from the catalog are consistent with those gained from analytic models using only low order moments of it. 


\section{References}

1. G. Cleghorn, Orbital Debris (Washington, National Research Council, 1995), Figs. 4-4 and 4-9.

2. D. McKnight, et. al., "Fragmentation Algorithms for Strategic and Theater Targets (FASTT) Empirical Breakup Model," DNA-Kaman January 1994.

(

3. D. Kessler, "Collisional Cascading: The Limits of Population Growth in Low Earth Orbit," Adv. Space Res, Vol. 11, 1991, p. (12)63.

4. D. Kessler and B. Cour-Palais, "Collision Frequency of Artificial Satellites: The Creation of a Debris Belt," Journal of Geophysical Research, Vol. 83, 1 June 1978, p. 2639. 5. G. Canavan, "Effect of Impactor area on Collision Rate Estimates," Los Alamos report, August
1966.

6. G. Canavan and O. Judd," Analytic Estimates of Space Debris Growth Rates," Los Alamos National Laboratory report LA-UR-95-3588, November 1995.

7. D. Kessler, "Collisional Cascading: The Limits of Population Growth in Low Earth Orbit," op. cit.

\section{DISCLAIMER}

This report was prepared as an account of work sponsored by an agency of the United States Government. Neither the United States Government nor any agency thereof, nor any of their employees, makes any warranty, express or implied, or assumes any legal liability or responsibility for the accuracy, completeness, or usefulness of any information, apparatus, product, or process disclosed, or represents that its use would not infringe privately owned rights. Reference herein to any specific commercial product, process, or service by trade name, trademark, manufacturer, or otherwise does not necessarily constitute or imply its endorsement, recommanufacturer, or otherwise doe United States Government or any agency thereof. The views and opinions of authors expressed herein do not necessarily state or reflect those of the United States Government or any agency thereof. 


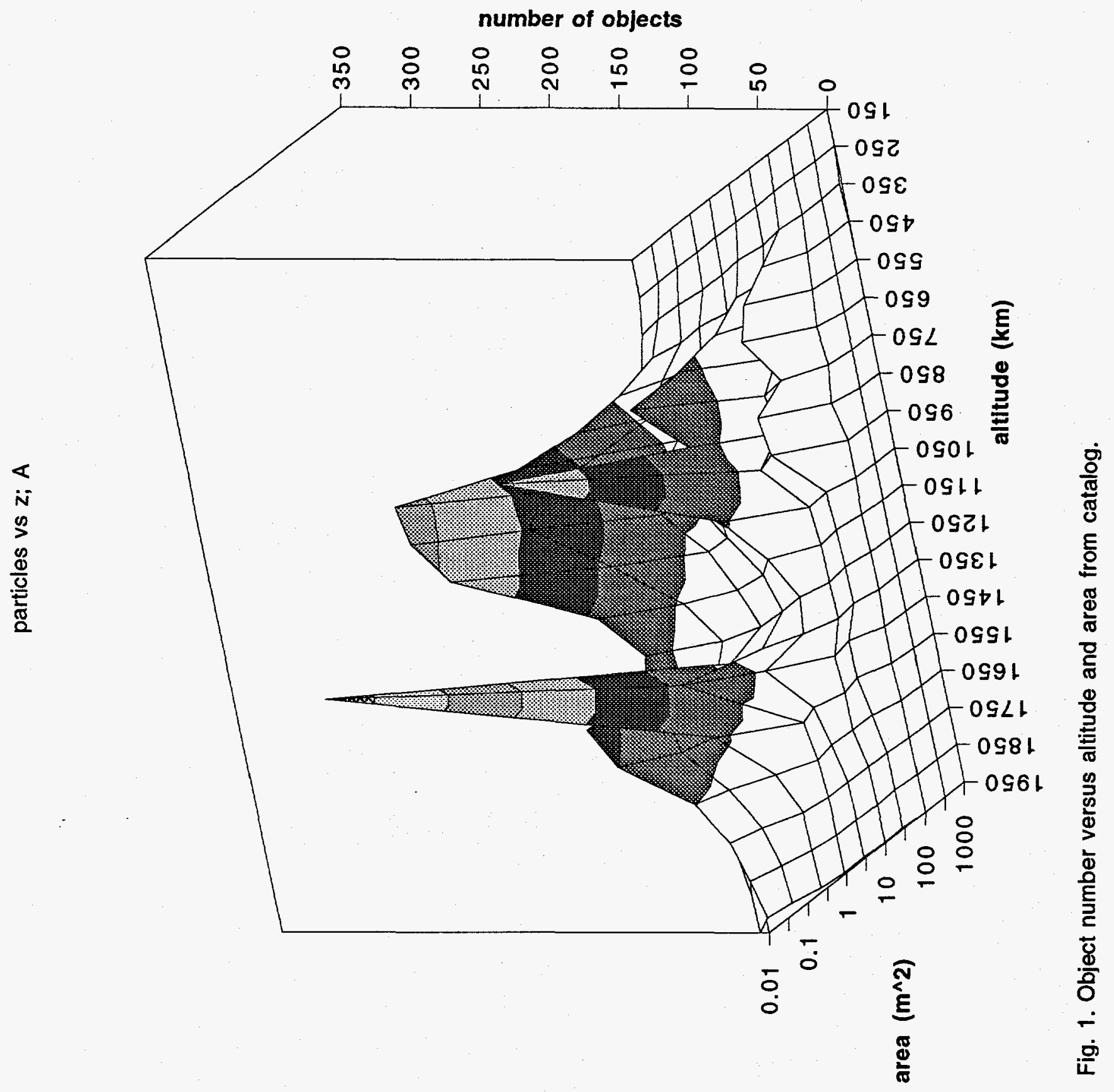




\section{particle number (/100 km bin)}

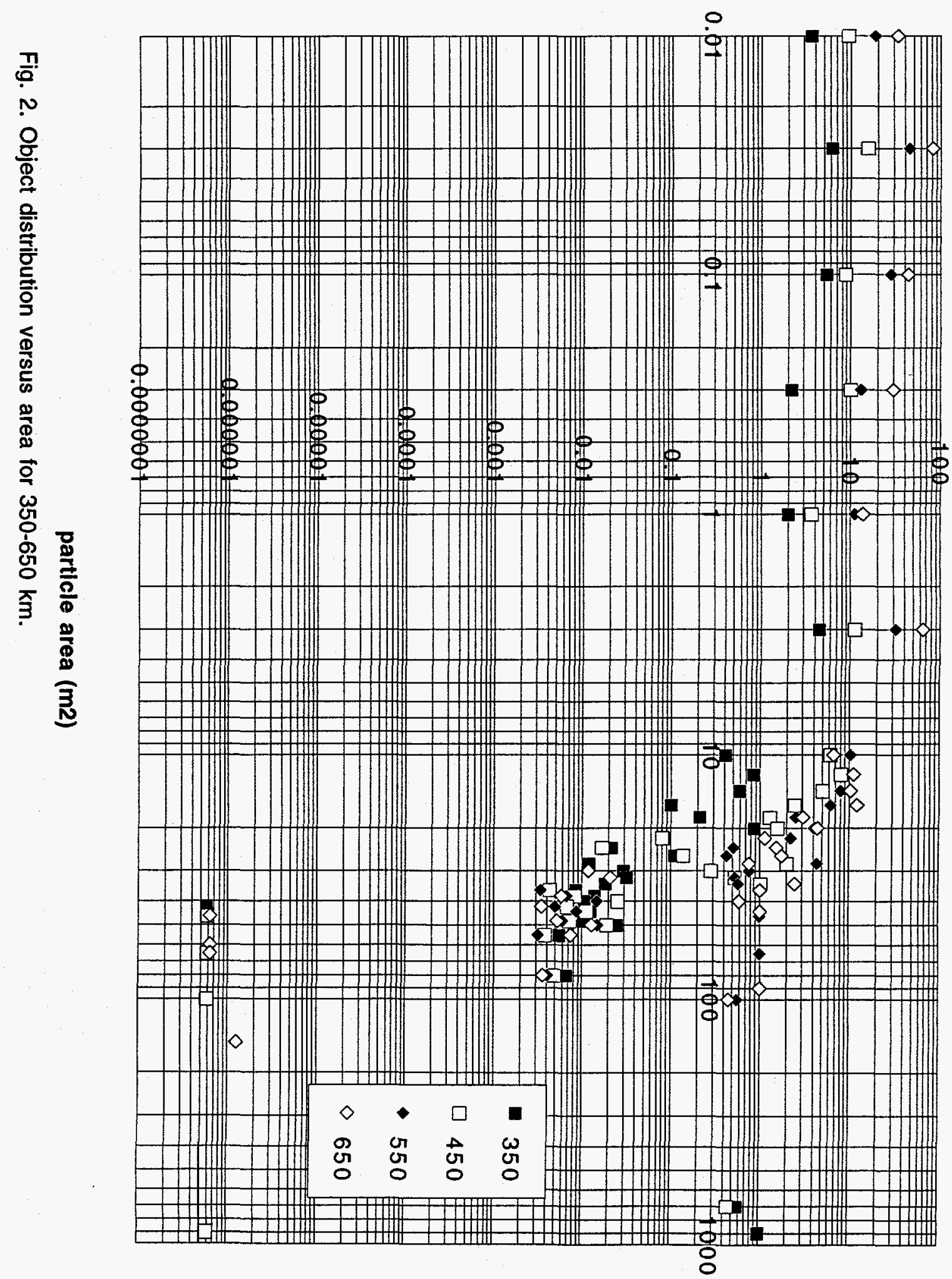




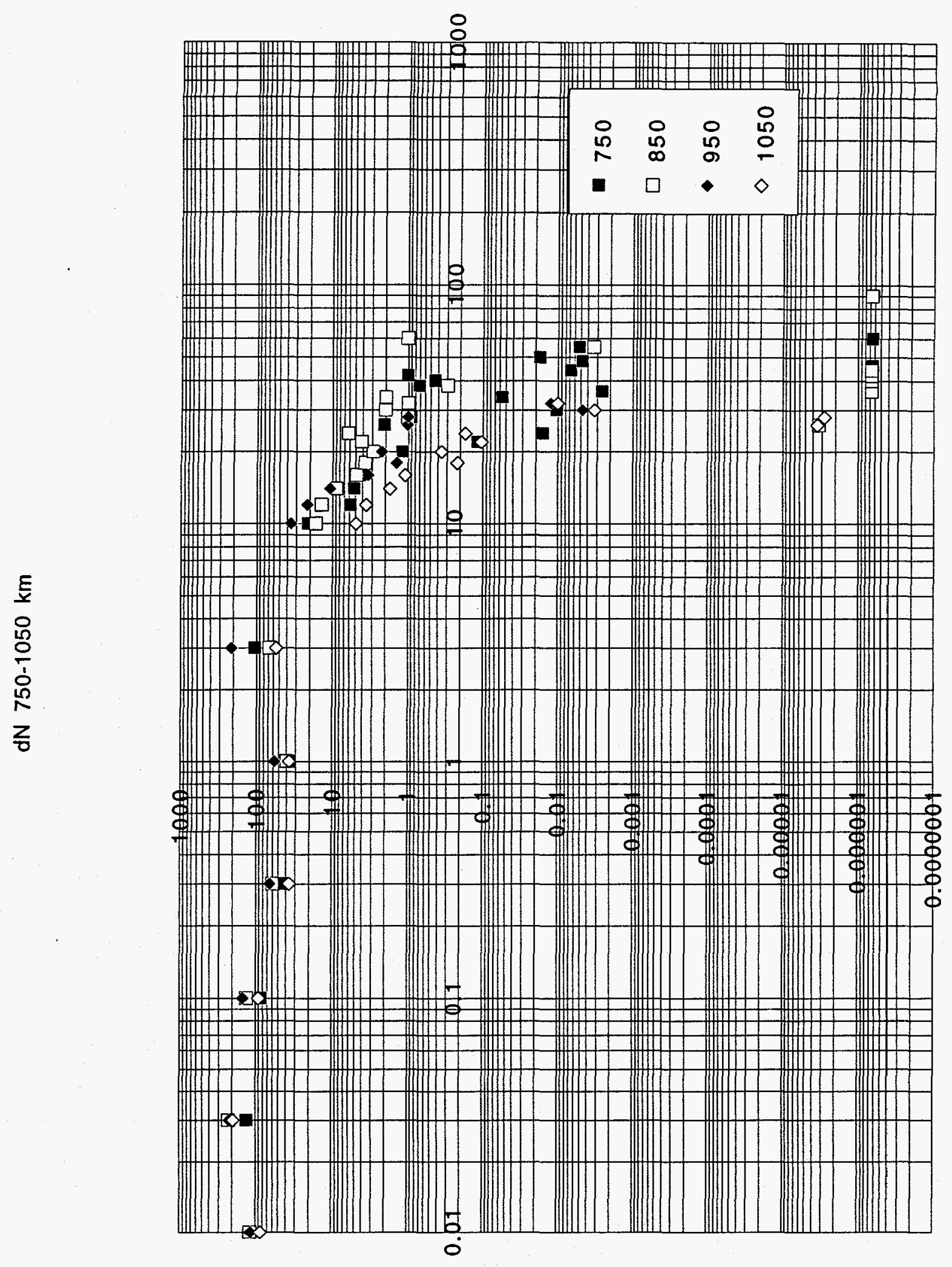

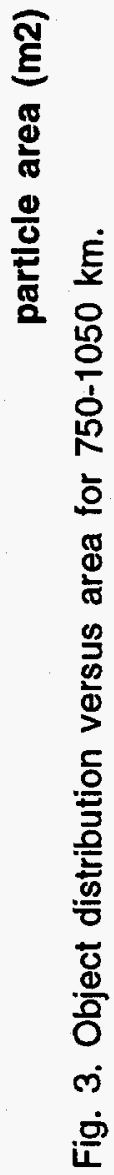

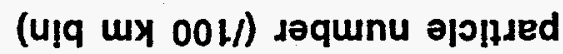


objects per $100 \mathrm{~km}$ bln per area

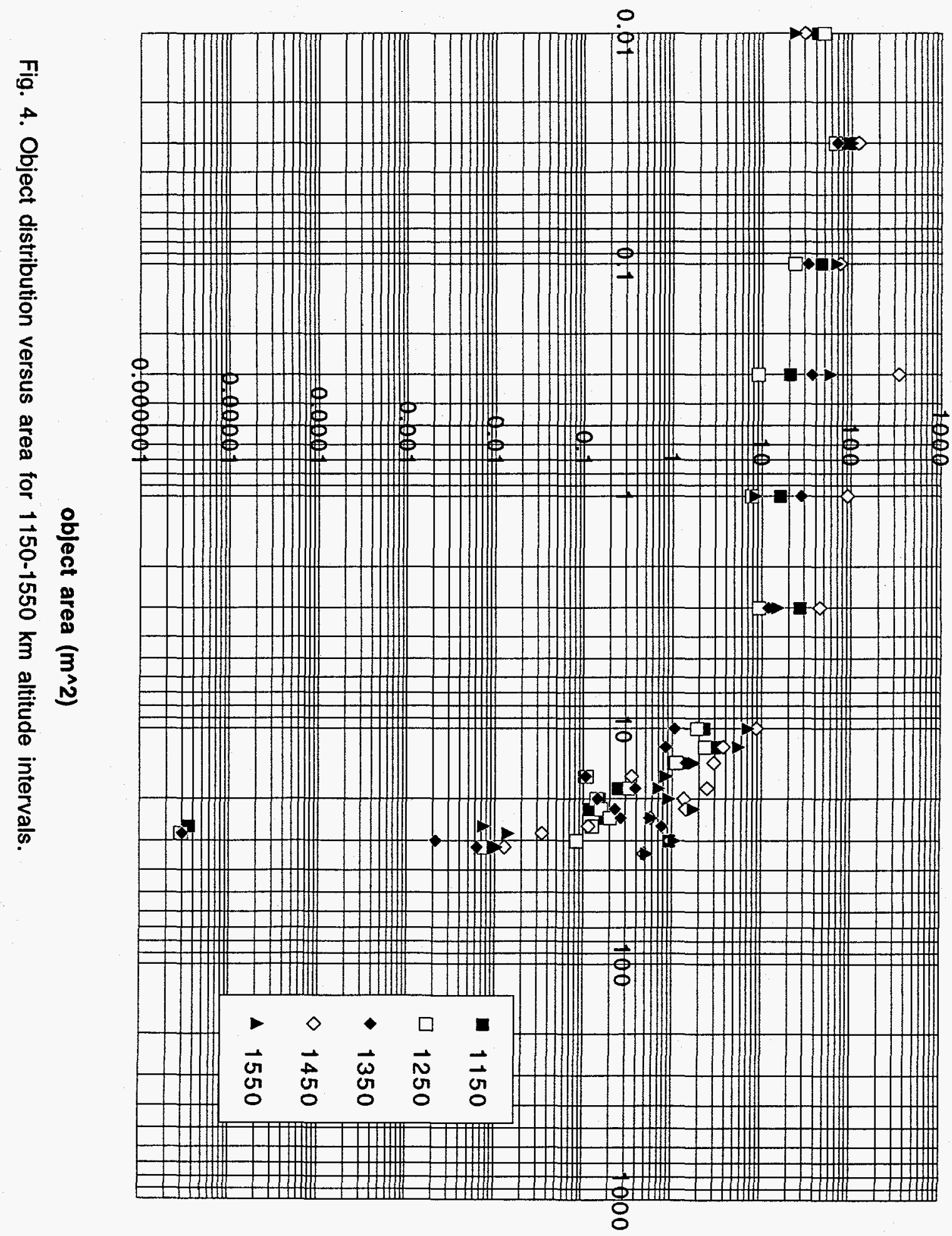




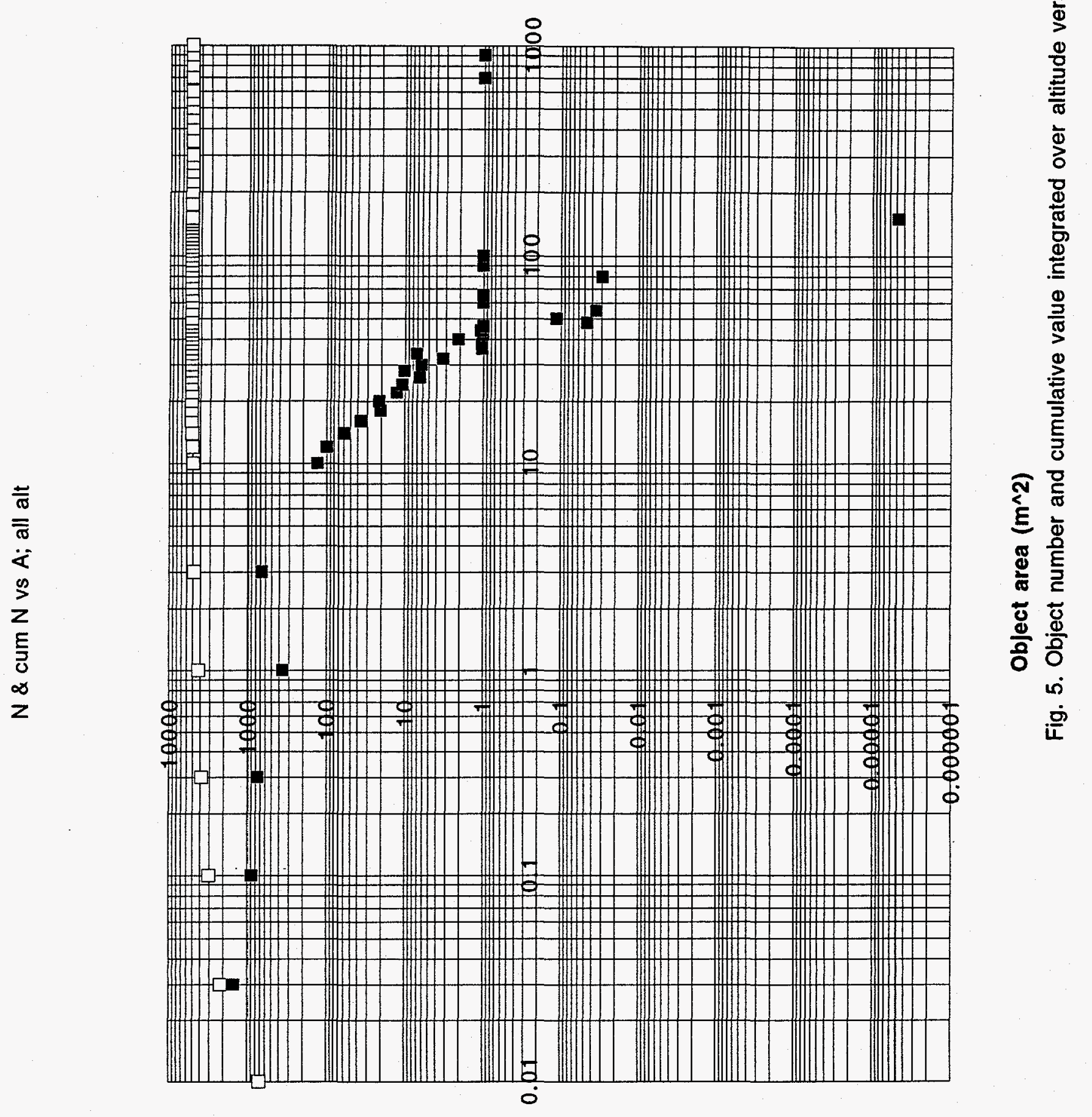

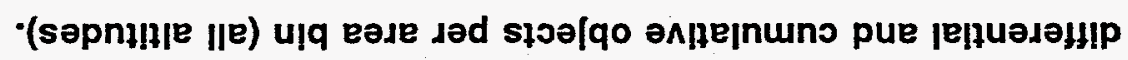


differential and cumulative area $\left(m^{\wedge} 2\right)$

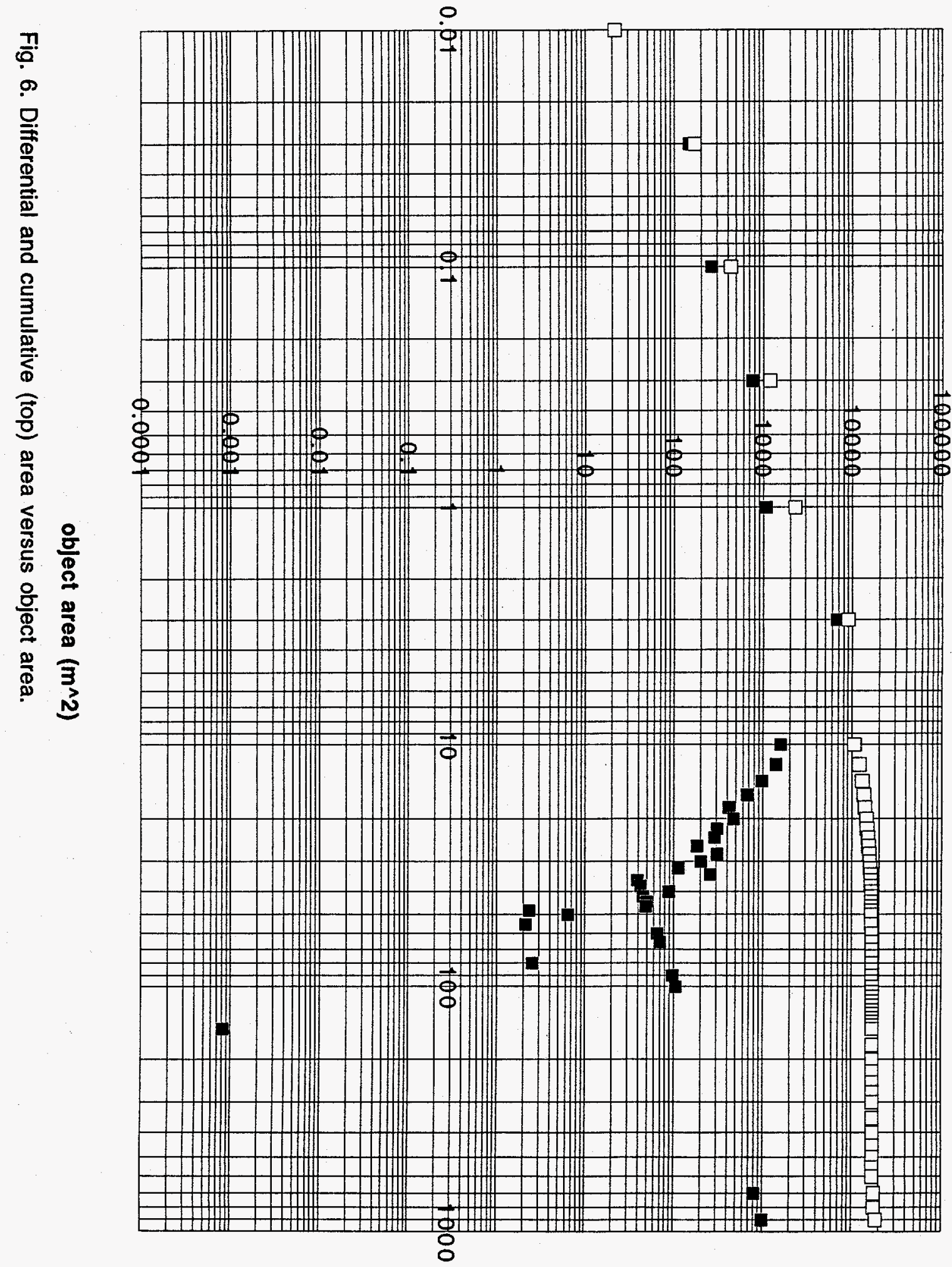

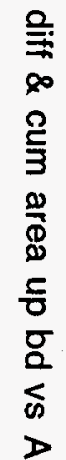




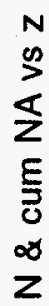

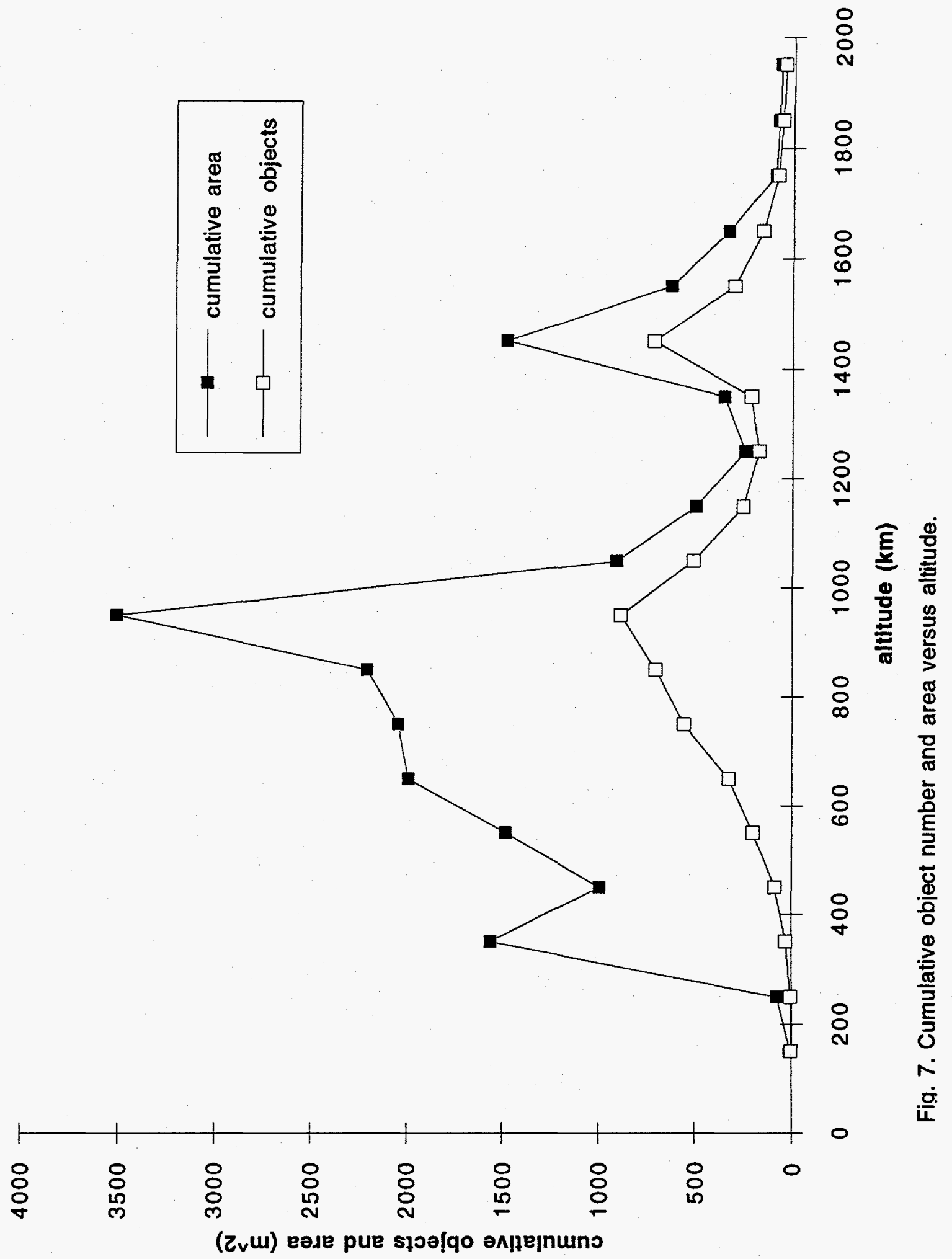




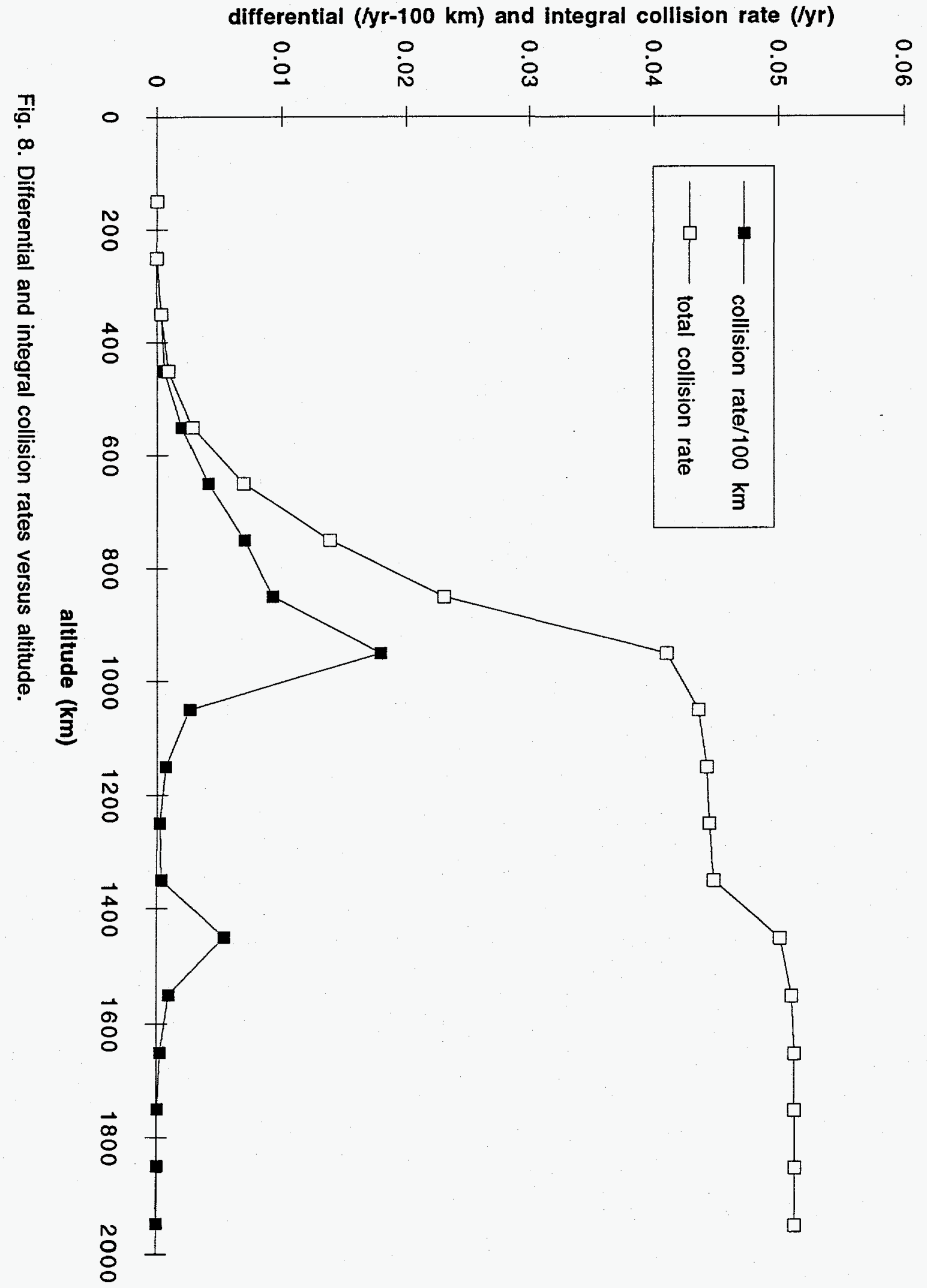




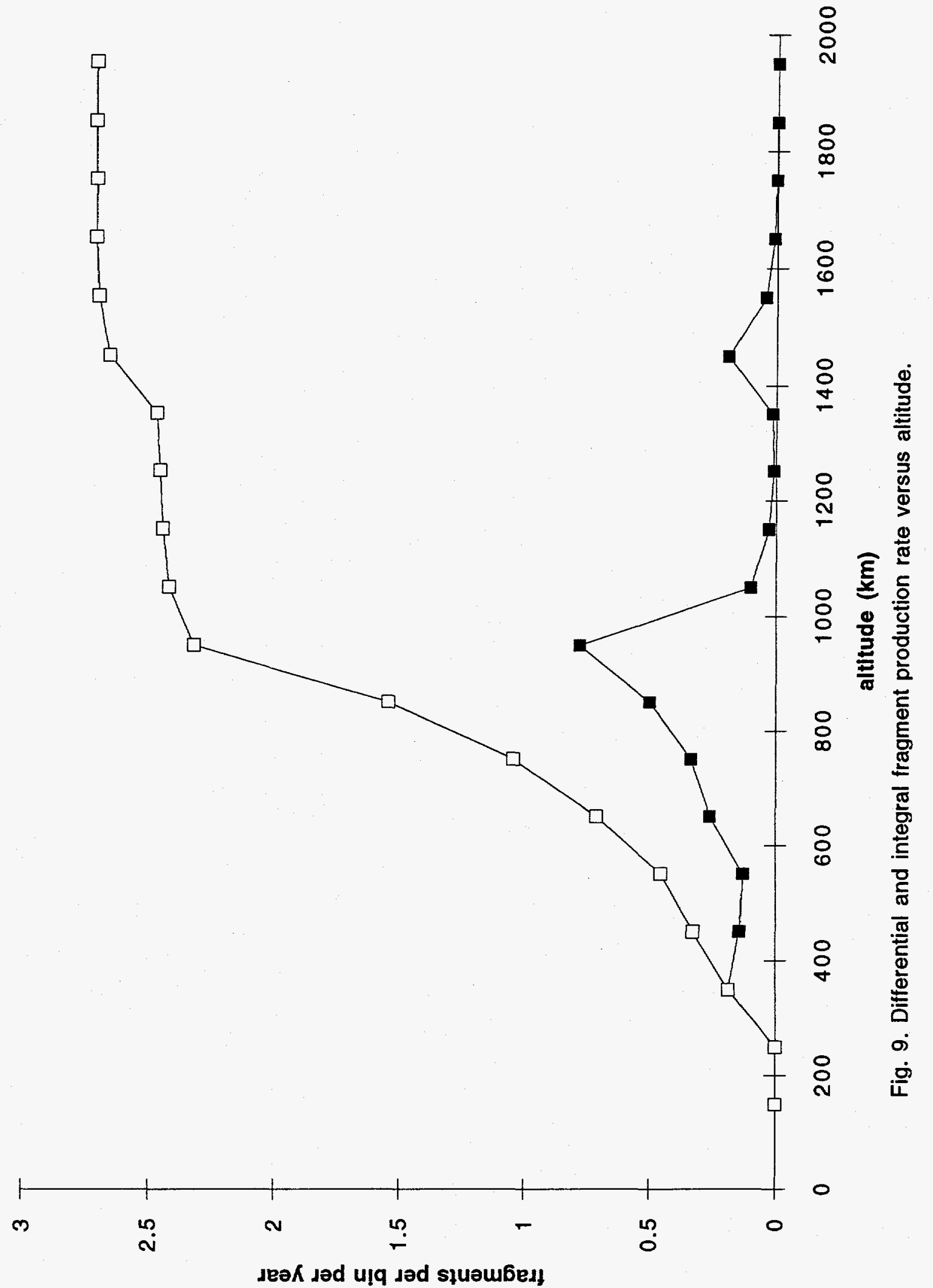




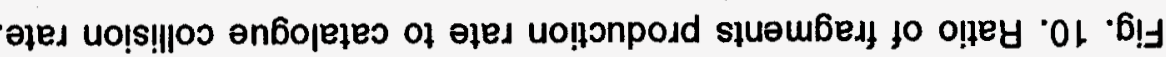
(uy) әpni|l|e

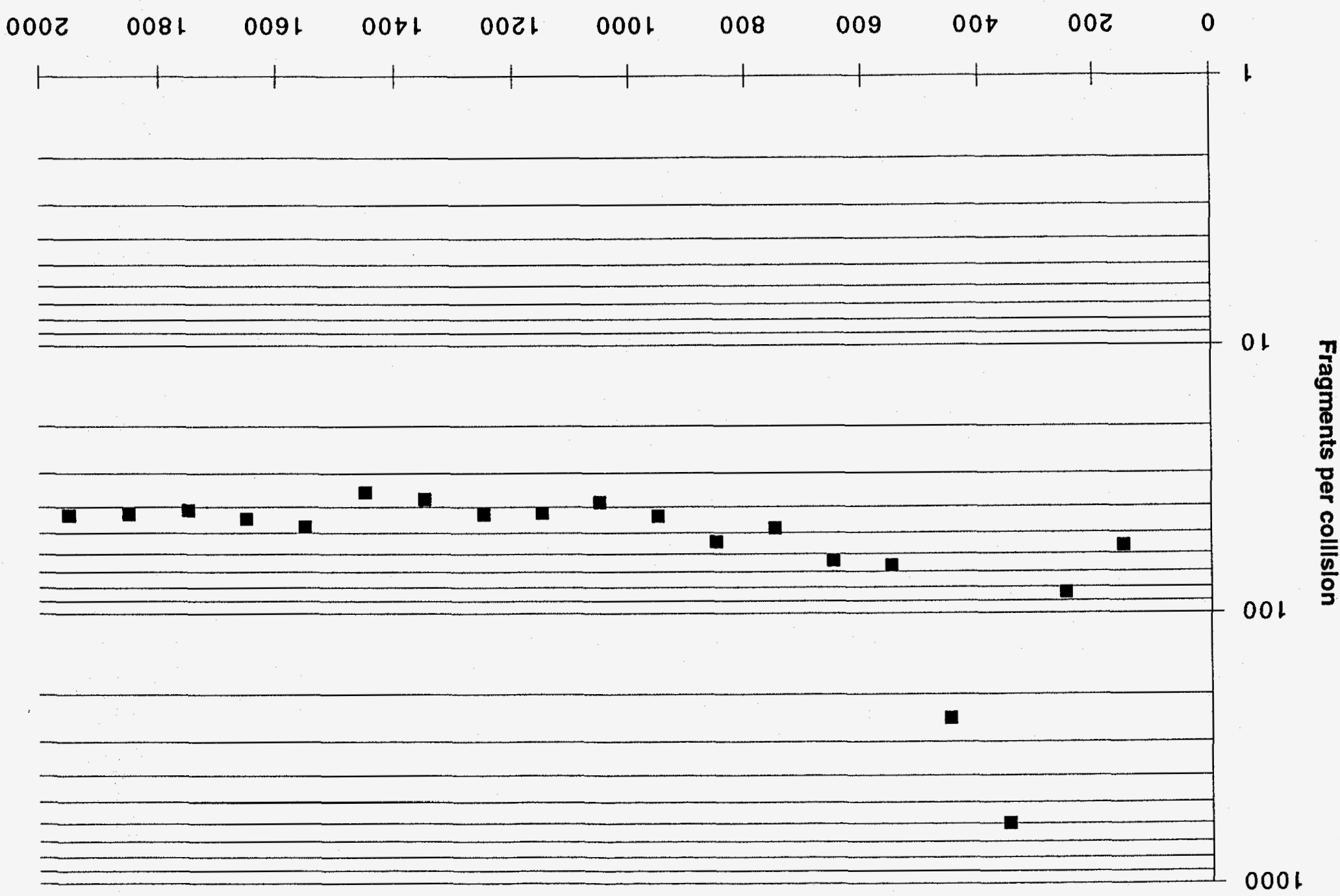




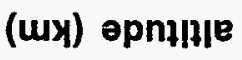

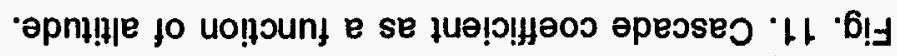

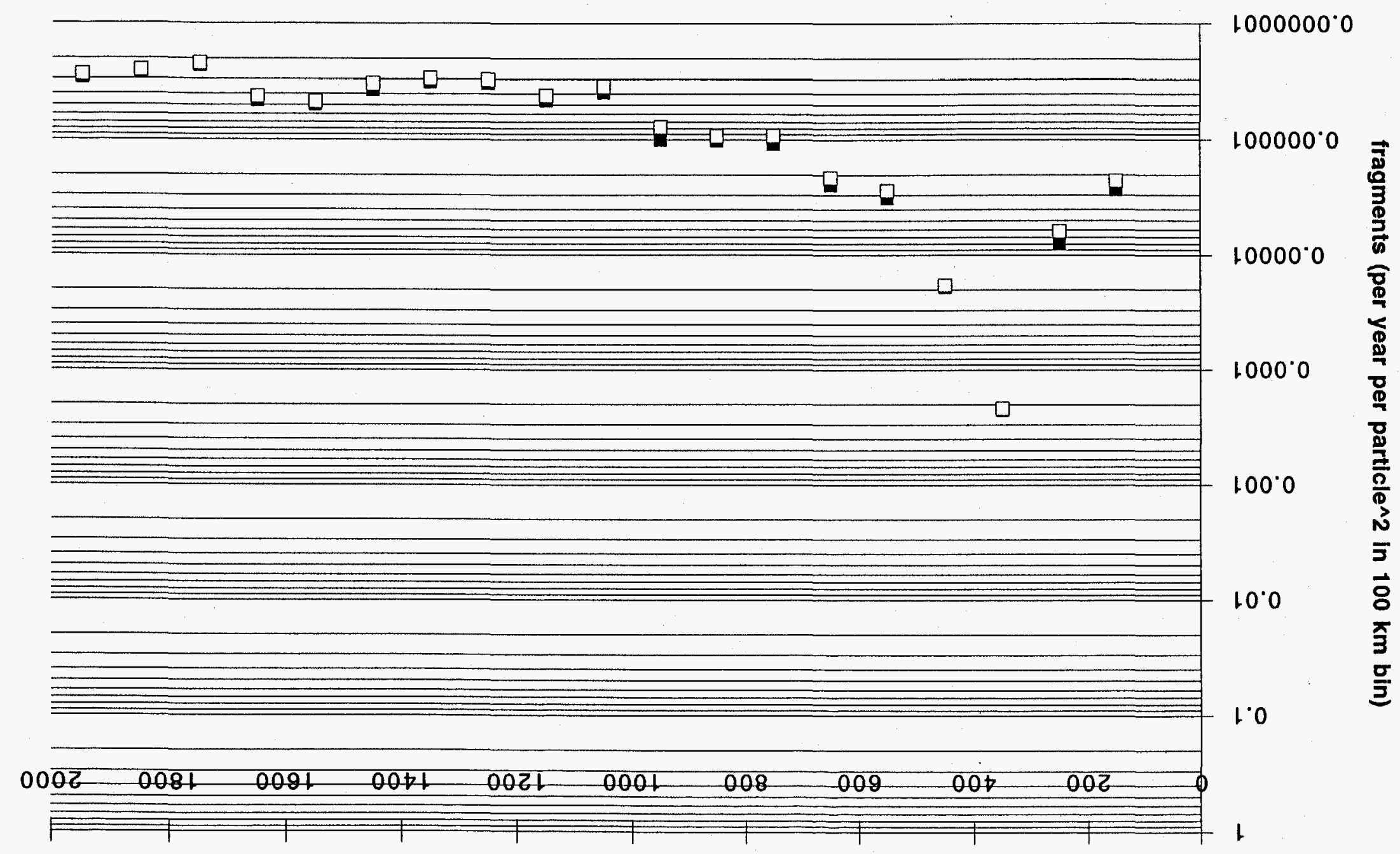

